\title{
Evaluation of the effects of colostrum replacer supplementation of the milk replacer ration on the occurrence of disease, antibiotic therapy, and performance of pre-weaned dairy calves
}

\author{
Manuel F. Chamorro, ${ }^{* 1}$ Natalia Cernicchiaro, $†$ and Deborah M. Haines ${ }^{*} \ddagger$ \\ *Department of Clinical Research, The Saskatoon Colostrum Company Ltd., Saskatoon, Canada S7K 6A2 \\ †Department of Diagnostic Medicine and Pathobiology, College of Veterinary Medicine, Kansas State University, Manhattan 66506 \\ ‡Department of Veterinary Microbiology, Western College of Veterinary Medicine, University of Saskatchewan, Saskatoon, Canada S7K 6A2
}

\begin{abstract}
The objective of this study was to evaluate the effects of colostrum supplementation of the milk replacer ration on disease occurrence, antibiotic therapy, and performance of pre-weaned dairy calves with adequate transfer of passive immunity. Two hundred and two 1-d-old Holstein dairy calves were assigned to 1 of 2 groups after arrival to a dairy calf rearing facility. Calves assigned to the control group $(\mathrm{n}=100)$ received milk replacer (28\% crude protein and $20 \%$ crude fat) without colostrum inclusion twice daily. Calves assigned to the treatment group $(\mathrm{n}=102)$ received 150 $\mathrm{g}$ of supplemental colostrum replacer powder added to their milk replacer twice daily for the first $14 \mathrm{~d}$ of life. Before group assignment, serum samples were collected from all calves to confirm transfer of passive immunity. Calves were evaluated daily until weaning ( $56 \mathrm{~d}$ of life) for signs of clinical disease as well as any treatment with antibiotics. Presentation of clinical disease and antibiotic treatment was recorded daily by personnel blinded to treatment allocation. Adequate transfer of passive immunity was confirmed in all calves at the start of the study and mean serum IgG values were similar among calves from treatment and control groups. The odds ratios of having abnormal feces and abnormal respiration during the pre-weaning period for calves from the treatment group were 0.15 and 0.46 the odds ratios of calves from the control group, respectively. The odds ratios of receiving antibiotic therapy during the preweaning period for calves from the treatment group were 0.09 the odds ratios of calves from the control group. Mean body weight and average daily gain at weaning were not significantly different among calves from the treatment and control groups. Colostrum replacer supplementation of the milk replacer ration was
\end{abstract}

Received June 22, 2016.

Accepted October 17, 2016

${ }^{1}$ Corresponding author: mchamorr@vet.k-state.edu effective in reducing antibiotic therapy and occurrence of disease during the pre-weaning period.

Key words: antibiotic, calf, colostrum, immunoglobulin $\mathrm{G}$

\section{INTRODUCTION}

Recently the livestock industry has been encouraged to reduce the use of antibiotics in food-producing animals. Concerns about the development of livestockorigin bacteria with resistance to antimicrobials used in human medicine have arisen (Silbergeld et al., 2008; Cheng et al., 2014); however, in livestock operations, morbidity and mortality due to infectious diseases is still a common cause of economic losses to producers (House, 1978; Pinchak et al., 2004; Windeyer et al., 2014). Regulatory agencies in the United States and several European countries have limited the number of antimicrobials allowed for therapeutic and preventive use in food-producing animals, and restriction of the currently available antibiotics is likely to increase in the near future (US Food and Drug Administration, 2010, 2015; Chantziaras et al., 2014). Additionally, the development of new antimicrobials for livestock species has been negligible in recent decades (Cheng et al., 2014). Therefore, developing alternatives to antibiotics for minimizing losses associated with infectious diseases is an evident need of the livestock industry.

Bovine colostrum has been used effectively in human medicine as an alternative or adjunct therapy to antimicrobials in the treatment of infectious diseases of the gastrointestinal tract (Steele et al., 2013). Antibacterial and antiviral properties of components of bovine colostrum such as lactoferrin and pro-inflammatory cytokines have been described (Hagiwara et al., 2000; Furlund et al., 2012). Specific antibodies against infectious pathogens contained in maternal colostrum provide protection to the newborn calf during the first months of life and are important predictors of health and performance for beef and dairy calves (Dewell et al., 2006; NAHMS, 2007; Windeyer et al., 2014). Ad- 
ditionally, results from some studies indicate that continuous administration of colostrum after gut closure ( $>24 \mathrm{~h}$ of age) could benefit overall calf health during the pre-weaning period (Snodgrass et al., 1982; Berge et al., 2009). Researchers from one study suggested that although continuous administration of colostrum to calves after $24 \mathrm{~h}$ of life did not result in further absorption of $\operatorname{IgG}$, the immunoglobulins and other immune factors present in colostrum prevented infection caused by enteric viruses and bacteria (Snodgrass et al., 1982). In another study, a low dose (70 g) of colostrum replacer was used to supplement the milk replacer ration of pre-weaned dairy calves for a short term (first $14 \mathrm{~d}$ of life). Results of this study demonstrated a reduced number of days with diarrhea and a reduced need for antibiotic therapy in calves with partial or complete failure in the transfer of passive immunity (Berge et al., 2009). However, scientific studies evaluating the effects of long-term, high-dose supplementation of colostrum replacers to dairy calves with adequate transfer of passive immunity and its effects on incidence of disease, antibiotic treatment, and performance remain limited.

The objective of the present study was to determine the effects of a supplemental dose of $150 \mathrm{~g}$ of colostrum replacer in the milk replacer ration for the first $14 \mathrm{~d}$ of life on the occurrence of disease, administration of antibiotic therapy, and performance (BW and ADG) parameters of pre-weaned dairy calves with adequate transfer of passive immunity.

\section{MATERIALS AND METHODS}

\section{Experimental Design and Sample Collection}

A convenience sample of 202 1-d-old Holstein dairy heifers with adequate transfer of passive immunity (only calves with serum IgG $>10 \mathrm{~g} / \mathrm{L}$ were included in the study) was used in this study. Study calves were born between June 1 and August 1, 2014. The sample size used in the present study was calculated based on the assumptions from previous studies with a similar scope (Berge et al., 2005, 2009). Calves originated from 10 different dairy farms located in Westphalia, Michigan. All farms were located within a 10-mile radius of the calf rearing facility. Herd size ranged from 80 to 250 milking cows. The predominant breed in all farms was Holstein. Calves were separated from their dams immediately after birth and received $4 \mathrm{~L}$ of maternal colostrum fed according to each farm's colostrum management protocol. Only 8 of the farms routinely dipped navels in newborn calves using a variety of povidone iodine and chlorhexidine products. The following day after colostrum feeding, calves were transported to a calf rearing facility located in the same area. This calf rearing facility provides service to 10 local dairy farms and maintains an approximate number of 1,200 dairy heifers from $1 \mathrm{~d}$ to 20 mo of age. Calves are received at $1 \mathrm{~d}$ of age and placed in individual sand-bedded hutches that are separated approximately by $1 \mathrm{~m}$ distance between each other. The bedding material is changed weekly. Between uses, calf hutches are thoroughly cleaned and disinfected with a commercial disinfectant (Virkon S, Chemorus, Wilmington, DE) and left empty for $2 \mathrm{wk}$ before re-use.

Serum samples $(10 \mathrm{~mL})$ were collected upon arrival to the calf rearing facility (d 0 ) from all calves for evaluation of serum IgG by single radial immunodiffusion. Thirteen calves did not meet the selection criteria of adequate passive transfer and were separated from the study and moved to another section of the facility. After arrival, all calves were randomly assigned to 1 of 2 different treatments using a random number generator (GraphPad, La Jolla, CA), and housed in individual calf hutches for the duration of the study. Calves fed milk replacer without colostrum replacer supplementation (control group; $\mathrm{n}=100$ ), received 454 $\mathrm{g}$ of a commercial milk replacer $(28 \% \mathrm{CP}, 20 \%$ crude fat; Land O'Lakes Milk Replacer, Shoreview, MN), mixed according to label directions to a final volume of $3 \mathrm{~L}$, twice daily until weaning (d 56). Calves fed milk replacer supplemented with colostrum replacer powder (treatment group; $\mathrm{n}=102$ ), received $150 \mathrm{~g}$ of dried bovine colostrum powder containing $32 \mathrm{~g}$ of $\operatorname{IgG}(>40 \%$ CP, $>20 \%$ crude fat; Calf's Choice Total Hi-Cal, The Saskatoon Colostrum Co. Ltd., Saskatchewan, Canada) added to $304 \mathrm{~g}$ of Land O'Lakes Milk Replacer powder and then mixed according to label directions to a final volume of $3 \mathrm{~L}$, twice daily for the first $14 \mathrm{~d}$ of life. Following $14 \mathrm{~d}$, calves from the treatment group received the same milk replacer at the same ratio and frequency as calves from the control group until abrupt weaning (d 56). In addition to milk replacer, all calves had access to fresh water and fresh calf starter daily during the entire study period. Individual calf BW were obtained at d 0,14, and 56 and ADG were calculated among groups. All study protocols performed within this trial were reviewed and approved by the animal care committee of the Saskatoon Colostrum Company Ltd. and were conducted in accordance with the principles and specific guidelines presented in the Guidelines for the Care and Use of Agricultural Animals in Research and Teaching (FASS, 2010).

\section{Assessment of Health Parameters and Antibiotic Therapy}

All calves were evaluated daily for signs of abnormal respiration, depression, fecal consistency, umbilical 
structures, and enlarged joints. Daily, each calf was assigned 2 scores on a scale of 0 to 3 to characterize whether it had abnormal respiration or fecal consistency. Additionally, 1 score on scale 0 to 2 and 2 scores on a scale of 0 to 1 were assigned to characterize whether the calf had an abnormal mentation, umbilicus, or joints, respectively. Briefly, calves without clinical signs of disease were assigned a score of 0 , whereas those with severe clinical signs of disease were assigned a score of 1,2 , or 3 depending on the parameter. Respiratory scores were considered abnormal $(>0)$ if the calf had serous nasal discharge (1), mucous nasal discharge (2), or mucopurulent nasal discharge (3). Depression was classified as none (0) if the calf was bright, a score of (1) was assigned if the calf was quiet but responsive, and a score of (2) if the calf was lethargic and nonresponsive. Feces were subjectively evaluated for consistency and were classified as normal (0), pasty (1), runny (2), or severe diarrhea (3; Chamorro et al., 2016). Normal feces were defined as being at least $80 \%$ solid material and $20 \%$ liquid; pasty feces were defined as feces with $70 \%$ solid material and $30 \%$ liquid; runny feces were defined as feces with $50 \%$ solid material and $50 \%$ liquid; and severe diarrhea was defined as $100 \%$ liquid feces with or without blood or fibrin. Umbilical scores were classified as normal (0) when enlargement of clinical structures was absent, or a score of 1 if enlarged and painful. Joint scores were classified as normal (0) when no enlarged joints were observed, or a score of 1 was assigned when one or more joints were enlarged.

Calves were evaluated twice daily by trained personnel from the calf rearing facility blinded to treatment allocation. Antibiotic therapy was administered when abnormal clinical scores were detected. The clinical condition rendering therapy with antimicrobials was recorded. The type of antibiotic and the total number of doses of antibiotics were also recorded. Antibiotic therapy was administered to calves based on pre-established calf rearing facility veterinary protocols. Briefly, calves presenting abnormal depression scores $(>1)$ in combination with abnormal respiratory $(>1)$, fecal $(>1)$, umbilical $(>0)$, or joint scores $(>0)$ warranted administration of antimicrobials. One of 4 different antimicrobials (hydrochloride ceftiofur, enrofloxacin, florfenicol, and tulathromycin) were used in each case depending on the clinical condition. A new case was defined as the presence of abnormal clinical scores in any category after at least $3 \mathrm{~d}$ of absence of abnormal scores. An antibiotic therapy was defined as at least a singly treatment with a long-acting antibiotic approved for food animal use or at least 3-consecutive-day treatments with a short-acting antibiotic approved for food animal use.

\section{Single Radial Immunodiffusion}

Serum (diluted 1:4) was assayed for total IgG concentration by single radial immunodiffusion, as previously described (Chelack et al., 1993). Antiserum against bovine $\operatorname{IgG}(\mathrm{H}$ and $\mathrm{L}$ chain) was used (Jackson Laboratories Inc., West Grove, PA). Single radial immunodiffusion plates were prepared from $2 \%$ agarose containing $2.5 \%$ antiserum in phosphate buffered saline (PBS, $\mathrm{pH}=7.25)$. Standard curves $(1.06-8.5 \mathrm{~g} / \mathrm{L})$ were produced using duplicate samples of a bovine IgG serum calibrator (Midlands Bio Products Corp., Boone, IA). The validity of plates was assessed with a reference serum from the Center for Veterinary Biologics, Animal Poultry Health Inspection Service, USDA. All samples were tested in triplicate and incubated in a humid atmosphere at $25^{\circ} \mathrm{C}$ for 18 to $24 \mathrm{~h}$. Ring diameters were measured with a computer-assisted plate reader (The Binding Site, Birmingham, England) and the values in samples calculated using a program for linear analysis.

\section{Statistical Analysis}

Descriptive statistics (mean, median, SD, range) were computed for all outcome variables, by group (Tables 1 and 2).

Table 1. Descriptive statistics of serum IgG, BW, and ADG by group (CS vs. MR $)^{1}$

\begin{tabular}{llrrrrrr}
\hline Variable, unit & Treatment & $\mathrm{n}$ & Mean & Median & SD $^{2}$ & Minimum & Maximum \\
\hline Mean BW (d 0), kg & CS & 102 & 40.6 & 40.4 & 4.8 & 27.7 & 51.3 \\
Mean BW (d 14), kg & MR & 100 & 39.2 & 39.7 & 4.8 & 20.4 & 50.3 \\
Mean BW (d 56), kg & MR & 101 & 45.1 & 44.9 & 3.8 & 34.9 & 54.4 \\
& CS & 98 & 44.4 & 44.9 & 4.4 & 31.1 & 52.6 \\
IgG (d 0), g/L & MR & 101 & 80.3 & 79.4 & 8.4 & 50.8 & 101.2 \\
ADG & CS & 102 & 79.6 & 80.3 & 9.1 & 51.7 & 99.8 \\
(Birth to weaning), kg & MR & 100 & 25.2 & 24.8 & 9.4 & 8.6 & 52.7 \\
\hline
\end{tabular}

${ }^{1} \mathrm{CS}=$ dried bovine colostrum powder + milk replacer; $\mathrm{MR}=$ milk replacer. 
Table 2. Descriptive statistics of antibiotic treatment by group (CS vs. MR) ${ }^{1}$

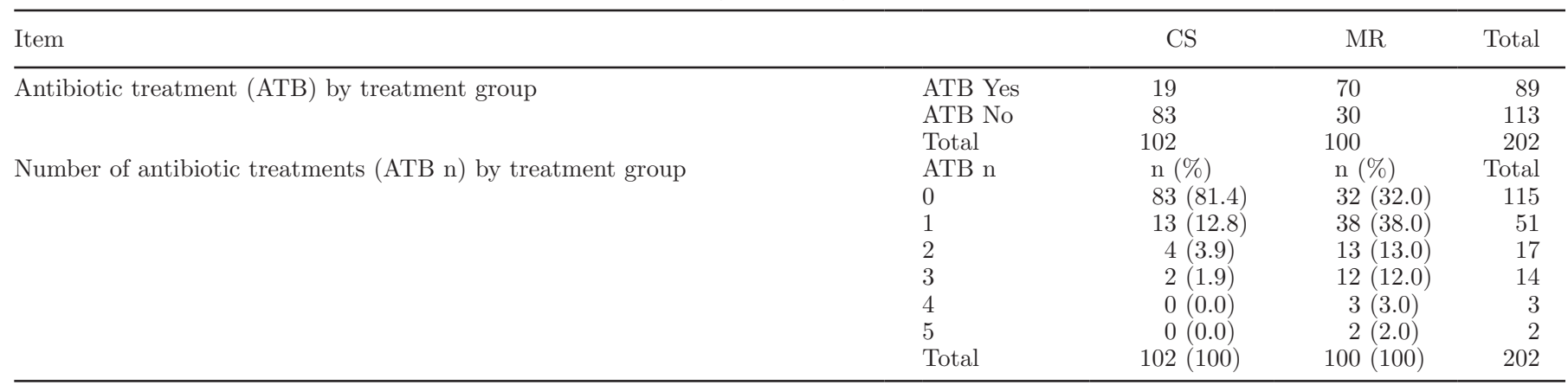

${ }^{1} \mathrm{CS}=$ dried bovine colostrum powder + milk replacer; $\mathrm{MR}=$ milk replacer.

The primary outcomes of interest included the occurrence of disease, specifically depicted by fecal, respiratory, depression, umbilical, and joint clinical scores, and the administration of antibiotic therapy. Secondary outcomes consisted of mortality, performance (BW and ADG from birth to weaning), and serum IgG levels.

\section{Sample Size}

The sample size was calculated based on the assumptions from previous studies with a similar scope (Berge et al., 2005, 2009). Briefly, the assumptions from these studies were the following: type I error $=0.10$, type II error $=0.10$, for detecting a difference of a mean of 5 morbid days for calves from unsupplemented and placebo groups compared with $3.5 \mathrm{~d}$ for calves from the colostrum supplement group. The assumption of sample standard deviation in these studies was $2 \mathrm{~d}$. The estimated sample size was inflated to allow for a $15 \%$ loss to follow-up. This corresponds to 30 to 36 animals per treatment group. As such, the enrollment of 101 animals per group in our study, under the same assumptions for the occurrence of disease (and as surrogate of antibiotic therapy), sufficed to detect differences of 0.8 to $1 \mathrm{~d}$ between treatment groups.

Clinical Scores. Associations between group (modeled as a dichotomous variable: treatment vs. control), study day (d 1 through 56), and a 2-way interaction between group and study day, with fecal scores, were determined using a generalized linear mixed model (GLMM) using the command Proc Glimmix in SAS 9.4 (SAS Institute Inc., Cary, NC). Models were fit using a multinomial distribution, cumulative logit link, restricted maximum likelihood estimation, and random intercepts for calf source (farm of origin), calf, and a first-order autoregressive covariance structure, to control for repeated measurements within calves over time (with equal spacing between measurements). Similar models were fit, separately, to assess associations of group, study day, and a 2-way interaction with respiratory and depression scores. Associations between group, study day, and a 2-way interaction with umbilical scores and joint scores (dichotomous outcomes) were fit in separate models, using GLMM with a binary distribution, logit link, restricted maximum pseudolikelihood, and random intercepts for calf source, calf, and a first-order autoregressive covariance structure.

Antibiotic Therapy. The association between group (treatment vs. control) with antibiotic therapy (modeled as a dichotomous outcome: 1 = antibiotic therapy vs. $0=$ no antibiotic therapy) was analyzed using GLMM. Models were fit using a binary distribution, logit link, restricted pseudo likelihood estimation, and a random intercept for calf source. Similarly, associations between group (treatment vs. control) with the number of doses of antibiotics received (modeled as a categorical (ordinal) outcome with 6 categories: 0 , $1,2,3,4$, or 5 doses) were modeled using GLMM with a multinomial distribution, cumulative logit link, restricted maximum likelihood estimation, and a random intercept for calf source.

Mortality, Performance Characteristics, and Serum Ig G. A GLMM with a binary distribution, logit link, restricted pseudo-likelihood, and a random intercept for calf source was fitted to evaluate associations between group (treatment vs. control) with mortality (dichotomous outcome with 2 categories: 1 = death, 0 $=$ alive). In addition, associations between group with continuous, normally distributed outcomes: serum IgG level on d 0 ( $\operatorname{IgG}$ ) and ADG from birth to weaning, were evaluated using GLMM, in separate models, using a Gaussian distribution, identity link, restricted maximum likelihood estimation, and a random intercept for calf source. For BW, associations between group, study day (d 0,14 , and 56), and a 2-way interaction between group and study day (modeled as a continuous outcome: recorded individual $\mathrm{BW}$ in $\mathrm{kg}$ ), were fit using a GLMM, as described above, and an additional random intercept for calf and an unstructured covariance structure, to account for repeated measures of multiple BW 
measurements recorded on study d 0, 14, and 56 (with unequal spacing between measurements).

For continuous outcomes, model-adjusted means and their $95 \%$ confidence intervals were computed, whereas for categorical outcomes, model-adjusted mean probabilities, odds ratios, and their $95 \%$ confidence intervals were estimated. For all analyses, values of $P<0.05$ were considered statistically significant.

Residual Diagnostics. Diagnostics of residuals from the final mixed-effects models (GLMM) included the evaluation of the predicted values or BLUP of the random variables in the model (e.g., calf source), and standardized, Pearson, and deviance residuals for observations at the lower level, depending on the distribution of the data. Normal probability plots and tests for normality (i.e., Shapiro-Wilk and Anderson-Darling) were examined to assess the normality assumption of the BLUP and general model fit. Graphical indication of departures from normality or statistically significant $(P<0.05)$ Shapiro-Wilk or Anderson-Darling statistics was used as a criterion to indicate lack of fit. In addition, residual plots for the lowest level units were visually examined to identify potential outliers and observations with undue influence on the model. Model assumptions (homoscedasticity and normality for continuous outcomes) and goodness of fit (HosmerLemeshow goodness of fit test for dichotomous, binary outcomes) were also assessed. For multinomial ordinal regression models, the proportional odds assumption was tested using a likelihood ratio test of proportionality of odds.

\section{RESULTS}

\section{Clinical Scores}

One calf from the treatment group and 2 calves from the control group died during the study period. One calf (control group) was euthanized due to the presence of a cleft palate and the other 2 calves (control group $=1$; treatment group $=1$ ) were euthanized due to bilateral abdominal distention that did not respond to specific therapy; however, we did not observe differences between groups (treatment group vs. control group) on mortality $(P=0.98)$. From the group of 202 study calves, 93 (treatment group $=20$; control group $=73$ ) presented abnormal depression scores $(\geq 1)$ at least once, 69 (treatment group $=9$; control group $=$ 60) presented abnormal fecal consistency scores $(\geq 1)$ at least once, 24 (treatment group $=11$; control group $=13)$ presented abnormal respiratory scores $(\geq 1)$ at least once, 11 (treatment group $=3$; control group $=8$ ) presented abnormal umbilical scores at least once, and
1 (treatment group $=1$; control group $=0)$ presented abnormal joint scores. The effect of group (treatment vs. control) was associated $(P<0.03)$ with the odds of a calf developing abnormal fecal consistency scores $(\geq 1)$, abnormal respiratory scores $(\geq 1)$, abnormal depression scores $(\geq 1)$, and abnormal umbilical scores (Table 3 ). Briefly, for calves from the treatment group, the odds of having abnormal fecal, respiratory, depression, and umbilical scores were $0.15,0.46,0.21$, and 0.18 times, respectively, the odds of control calves. Therefore, calves from the treatment group were less likely to experience abnormal fecal, respiratory, depression, and umbilical scores compared with control calves (Table 4).

\section{Antibiotic Therapy}

The effect of group (treatment vs. control) was associated $(P<0.01)$ with the probability of a calf receiving antibiotic therapy. The probability of receiving antibiotic therapy for calves from the treatment group was $18.8 \%$, whereas the probability of receiving antibiotic therapy for calves from the control group was $76.5 \%$. Therefore, calves from the treatment group tended to have a lower likelihood (odds ratio $=0.07$ ) of being treated with antibiotics compared with control calves (Table 4). Of all calves that received antibiotic therapy, 50 (treatment group $=12$; control group $=38$ ) received a single dose of antibiotics, 17 (treatment group $=4$; control group $=13$ ) received 2 doses, 14 (treatment group $=2$; control group $=12$ ) received 3 doses, and 6 (treatment group $=1$; control group $=5$ ) received more than 3 doses of antibiotics during the study period. For calves from the treatment group, the odds of receiving 1 or more doses of antibiotics were 0.09 or $89 \%$ lower compared with the odds of calves from the control group, and this association was statistically significant. Thus, calves from the treatment group had a lower likelihood of receiving more antibiotic treatments than control calves (Table 4).

\section{Serum $\lg G$}

At d 0 (arrival), all calves had adequate transfer of passive immunity (serum IgG $>10 \mathrm{~g} / \mathrm{L}$ ) and most calves had excellent transfer of passive immunity (serum IgG $>15 \mathrm{~g} / \mathrm{L}$ at $24 \mathrm{~h}$; Table 3; NAHMS, 2007). The mean level of IgG in the serum of calves from both the treatment and control groups was not different (treatment group $=23.6 ;$ control group $=22.8 ; P=0.51$, Table 5) . Serum IgG at arrival did not have a significant effect on mortality, presentation of abnormal clinical scores, or antibiotic therapy between treatment and control calves. 
COLOSTRUM REPLACER ON DISEASE AND USE OF ANTIBIOTICS

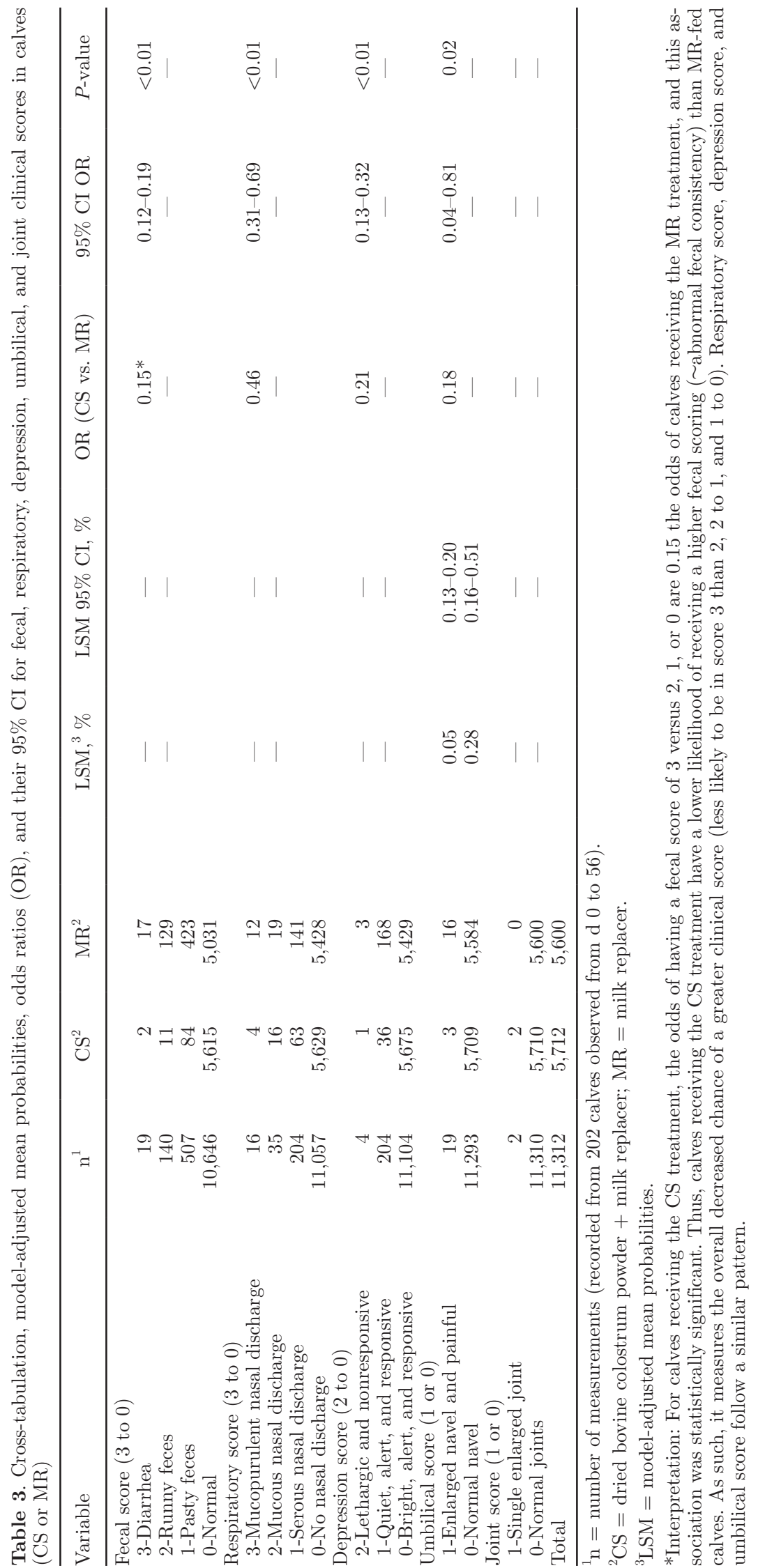


Table 4. Associations between group (CS vs. MR) ${ }^{1}$ and antibiotic treatment

\begin{tabular}{llcrcrc}
\hline Variable & Treatment & LSM, $\%$ & LSM $^{2}$ 95\% CI, \% & P-value & OR & OR 95\% CI \\
\hline Antibiotic treatment & CS & 18.8 & $6.8-42.4$ & $<0.01$ & $0.07^{*}$ & $0.03-0.15$ \\
(Yes vs. no) & MR & 76.5 & $49.3-91.6$ & $<0.01$ & $0.09^{* *}$ & $0.04-0.17$ \\
Number of antibiotic treatments (5 to 0) & CS vs. MR & & & & $<0.01$ \\
\hline
\end{tabular}

${ }^{1} \mathrm{CS}=$ dried bovine colostrum powder + milk replacer; $\mathrm{MR}=$ milk replacer.

${ }^{2} \mathrm{LSM}=$ [model-adjusted mean probabilities for categorical outcomes (antibiotic therapy, number of antibiotic treatments)].

${ }^{3} \mathrm{OR}=$ odds ratio.

*For calves receiving the CS treatment, the odds of receiving antibiotic therapy were 0.07 the odds of calves receiving the MR treatment, and this association was statistically significant. Thus, CS calves had a lower likelihood of receiving antibiotic therapy than MR calves.

${ }^{* *}$ For calves receiving the CS treatment, the odds of receiving 5 treatments versus $4,3,2,1$, or none were 0.09 the odds of calves receiving the MR treatment, and this association was statistically significant. Thus, CS calves had a lower likelihood of receiving more antibiotic treatments than MR calves.

\section{Performance}

The effect of group was marginally associated $(P=$ $0.07)$, and study day was associated $(P<0.05)$, with mean BW from d 0 to 56. Mean BW increased in all calves toward the end of the study; however, we observed a difference in the mean BW between treatment and control calves at d 0 (40.6 vs. $39.2 \mathrm{~kg}$, for treatment and control calves, respectively; $P=0.04$ ). Additionally, the mean ADG of treatment and control calves from d 0 to 56 was not different $(P=0.18$, Table 5$)$.

\section{DISCUSSION}

Failure in the transfer of passive immunity is one of the most important risk factors and predictors of dairy calf morbidity and mortality (NAHMS, 2007; Windeyer et al., 2014). Results from a previous report where milk replacer was supplemented with colostrum replacer in pre-weaned dairy calves indicated that failure in the transfer of passive immunity was the most important predictor of diarrhea and antibiotic treatment (Berge et al., 2009); as a consequence, the emphasis for amelioration of neonatal disease has been on programs to improve colostrum management and levels of transfer of passive immunity. In contrast, in the present study the mean level of serum IgG at arrival to the calf rearing facility in calves from both treatment and control groups was well above the limit accepted for adequate transfer of passive immunity $(>10 \mathrm{~g} / \mathrm{L})$ and in fact was above the levels proposed to be consistent with "excellent" transfer of passive immunity $(15 \mathrm{mg} / \mathrm{dL}$; NAHMS, 2007). Under these conditions of successful transfer of passive immunity, mean serum IgG at arrival did not have a significant effect on mortality, presentation of abnormal clinical scores, or antibiotic therapy. This suggests that high levels of serum IgG after colostrum intake do not always provide complete protection against the broad spectrum of ubiquitous pathogens found in the environment of neonatal dairy calves. Additionally, results from previous studies have indicated a lack of association between IgG intake from colostrum and presentation of diarrhea in calves (KleinJöbstl et al., 2014; Meganck et al., 2015).

Mortality in this trial was low (1.48\%) possibly due to the high levels of serum IgG in all calves at the start of the study; however, many calves in the control group developed abnormally loose feces and several presented with respiratory abnormalities. This suggests that maternally derived immunity does not always protect against infectious challenges at the mucosal surface of the enteric and respiratory tracts. Additionally, pathogen overload or differences in the specificity of immunity transferred through maternal colostrum (or both) could have surpassed the protection provided by maternally derived specific antibodies and contributed to the presentation of clinical disease. A prevalence of calf diarrhea similar to the one reported in the present study has been previously reported in dairy operations (Windeyer et al., 2014; Meganck et al., 2015). Results

Table 5. Associations between group (CS vs. MR) $)^{1}$ IgG level on d 0, and ADG from birth to weaning

\begin{tabular}{llccc}
\hline Variable & Treatment & LSM, \% & LSM $^{2}$ 95\% CI, \% & $P$-value \\
\hline IgG & CS & 23.65 & $20.72-26.58$ & 0.51 \\
(d 0), g/L & MR & 22.83 & $19.80-25.87$ & \\
ADG & CS & 0.73 & $0.70-0.76$ & 0.17 \\
(Birth to weaning), $\mathrm{kg}$ & MR & 0.70 & $0.67-0.73$ & \\
\hline
\end{tabular}

${ }^{1} \mathrm{CS}=$ dried bovine colostrum powder + milk replacer; $\mathrm{MR}=$ milk replacer.

${ }^{2} \mathrm{LSM}=[$ model-adjusted means for continuous outcomes (IgG, ADG)]. 
from previous studies have also demonstrated that high variability in serum titers of specific antibodies in maternal-colostrum-fed calves could result in variation of the duration and level of protection against calf infectious agents (Chamorro et al., 2014; Pardon et al., 2015).

In this study, the post-gut-closure supplementation of the milk replacer ration with approximately $30 \%$ (150 $\mathrm{g}$ of $14 \%$ IgG powder) of a colostrum replacer twice daily during the first $14 \mathrm{~d}$ of life exerted a protective effect against the presentation of diarrhea (abnormal fecal scores), respiratory disease (abnormal respiratory scores), umbilical enlargement, and antibiotic therapy in dairy calves. Other researchers have reported reduced morbidity and mortality due to enteric disease in calves that receive milk or milk replacer supplemented with colostrum or plasma products (Snodgrass et al., 1982; Quigley and Wolfe, 2003; Parreño et al., 2010). Snodgrass et al. (1982) reported that dairy calves receiving whole milk supplemented with $10 \%$ of maternal colostrum from cows previously vaccinated against rotavirus had a delayed onset of diarrhea and reduced clinical disease compared with calves receiving whole milk or whole milk supplemented with $10 \%$ of pooled maternal colostrum. Parreño et al. (2010) reported 80\% prevention of diarrhea in 10 dairy calves that received pasteurized milk supplemented with $0.8 \%$ hyperimmune bovine colostrum (approximately $32 \mathrm{~mL}$ of hyperimmune bovine colostrum added to $2 \mathrm{~L}$ of pasteurized milk twice daily) during the first $14 \mathrm{~d}$ of life. Similarly, Quigley and Wolfe (2003) reported a 30\% reduction of diarrhea and reduction of the total number of days on antibiotic therapy in 40 dairy calves that received commercial milk replacer supplemented with spray-dried bovine plasma at $5 \%$ of the ration (approximately $23 \mathrm{~g}$ of spray-dried bovine plasma added to the milk replacer ration twice daily) during the first $42 \mathrm{~d}$ of life.

In another study, researchers reported that a supplementation rate of $70 \mathrm{~g}$ of $>14 \% \mathrm{IgG}$ powder reduced the relative risk of diarrhea in calves (relative risk $=0.61$ ). The authors of this study indicated that the presence of failure in the transfer of passive immunity increased the risk of diarrhea in nonsupplemented calves (relative risk $=1.07)$; however, the relative risk for respiratory disease was not different among supplemented and nonsupplemented calves (Berge et al., 2009). With respect to antibiotic therapy, researchers of the same study reported that supplemented calves had the lowest percentage of antimicrobial treatment days $(8.8 \%)$ compared with placebo and control calves (12.3 and 10.6\%, respectively). In the present study, $150 \mathrm{~g}$ of $>14 \% \mathrm{IgG}$ powder added to the milk replacer ration of calves with adequate transfer of passive immunity during the first $14 \mathrm{~d}$ of life reduced the risk of diarrhea, respiratory disease, and umbilical enlargement. Additionally, supplemented calves had a reduced total number of doses of antibiotics compared with controls. These results indicate that although lower supplementation rates of colostrum powder could be effective at reducing the risk of enteric disease and antibiotic therapy in dairy calves, a higher supplementation rate $(150 \mathrm{~g}$ of $>14 \%$ IgG powder) could reduce not only enteric disease but also respiratory and umbilical disease in addition to overall antibiotic therapy.

Local immunoglobulins as well as other immune and growth factors present in maternal colostrum such as lactoferrin, TNF- $\alpha$, epidermal growth factor, IL-6, and IL-1 $\beta$ could potentiate antiviral and bactericidal activities in the intestine and modulate the immune response and mucosal healing in neonates (Blum and Hammon, 2000; Arthington et al., 2002; Bagwe et al., 2015). The local effect of these factors present in the colostrum replacer product could have provided additional protection against enteric pathogens in supplemented calves. Reduction of enteric infections could have indirectly reduced infection of the upper respiratory tract and umbilicus by decreasing the risk of immunosuppression and by maintaining active and effective immune responses. Results from one study demonstrated an increased risk of respiratory disease in pre-weaned dairy calves that present other diseases during the first $2 \mathrm{wk}$ of life (Windeyer et al., 2014). Additionally, a positive significant association between the herd-level treatment risk of respiratory disease with the herd-level treatment risk of diarrhea has been reported in calves (Murray et al., 2016). The post-gut-closure intestinal translocation of smaller immunomodulatory molecules from the colostrum replacer such as cytokines that enhance general systemic immune responses against infection could offer another explanation of the overall decreased occurrence of disease observed in supplemented calves. Results from another study demonstrated that the total concentration and mRNA expression of cytokines such as IL-1 $\beta$, IL-6, TNF- $\alpha$, INF- $\gamma$, or IL-1 receptor antagonist were higher in bovine colostrum compared with milk (Hagiwara et al., 2000); however, additional research studies are necessary to completely understand the role of bovine colostrum's immunomodulatory molecules on intestinal and systemic immune responses and infection in pre-weaned dairy calves.

The use of antibiotics for the treatment of bovine respiratory disease and umbilical infection is a common practice among bovine practitioners (Shearer, 1986; Apley, 2015); however, the treatment of calf diarrhea with antimicrobials remains a controversial topic among veterinarians. The risk of bacteremia in diarrheic calves supports the use of antimicrobials in some cases (Fecteau et al., 1997); in contrast, the potential 
for causing disturbances on the intestinal microflora and increasing the risk of bacterial resistance discourages the use of antimicrobials in the treatment of calf diarrhea in other cases (Xie et al., 2013). In the present study, local and systemic protection provided by immunoglobulins and immunomodulatory molecules from the colostrum powder might have reduced the incidence of infection and therefore the need of antibiotic therapy in supplemented calves. Results from one study indicated that the presence of high concentrations of specific antibodies against Clostridium difficile toxins $\mathrm{A}$ and $\mathrm{B}$ contained in hyperimmune bovine colostrum was effective in preventing diarrhea in gnotobiotic piglets experimentally infected with Clostridium difficile (Sponseller et al., 2015).

Based on results from this and a previous study (Berge et al., 2009), no effect was found of group on mean BW and ADG. Higher mean birth weights could have influenced the differences in BW observed at $\mathrm{d} 0$ among calves from the treatment and control groups. The extra energy provided by the colostrum replacer (slightly higher in CP and fat compared with milk replacer) in calves from the treatment group was probably not sufficient to generate significant differences in $\mathrm{BW}$ or $\mathrm{ADG}$ when compared with control calves. The higher level of colostrum supplementation used in this study ( $150 \mathrm{~g}$ of $>14 \%$ IgG powder administered twice daily) in comparison to that used in a previous study (70 g of $>14 \%$ IgG powder administered twice daily; Berge et al., 2009) was expected to improve performance in treated calves; however, beneficial effects were only observed in health parameters and antibiotic therapy. Additionally, the level of colostrum replacer supplementation used in the present study may be cost prohibitive for producers based on current economic conditions (approximately US $\$ 12.00$ per calf per day of supplementation). Therefore, a higher supplementation dose of colostrum replacer powder might not necessarily result in higher mean BW and ADG at weaning but might improve overall calf health reducing both enteric and respiratory disease and leading to a greater reduction in the use of antibiotics in dairy calves.

It is important to mention that to minimize the chance of spurious results due to the multiplicity of outcomes tested, for our study we considered, a priori, primary outcomes (i.e., occurrence of disease and use of antibiotic therapy) and a limited number of secondary outcomes (e.g., mortality, performance characteristics). Even though all outcomes were analyzed separately, the risk of inflating type I error when assessing multiple outcomes is increased. Granting $P$-value adjustments can be attempted to reduce the chance of making type I errors; corrections for multiplicity are not always straightforward, and can also increase the chance of making type II errors, and make the interpretation of a large number of $P$-values challenging (Tyler et al., 2011). We expect that future research studies in this subject could help corroborate our findings.

\section{CONCLUSIONS}

Colostrum replacer products have been advocated as an alternative to prevent failure in the transfer of passive immunity in calves when availability of maternal colostrum is low or when quality of maternal colostrum is compromised due to low $\operatorname{IgG}$ levels or the presence of colostrum-borne pathogens (Chamorro et al., 2014). However, few studies have evaluated the ability of these products to decrease disease presentation and overall antibiotic therapy when used post gut-closure in dairy calves and no other studies have examined the effects in calves with adequate transfer of passive immunity. Based on results from this and a previous study (Berge et al., 2009), this dried-colostrum colostrum replacement product could be used as a supplement of the milk replacer diet to decrease the occurrence of disease and the associated need for antibiotic therapy in preweaned dairy calves irrespective of their status in the transfer of passive immunity.

\section{ACKNOWLEDGMENTS}

The authors thank Sonrise farms, and especially Deborah Feldpausch for the excellent collaboration and technical assistance with this project.

\section{REFERENCES}

Apley, M. D. 2015. Treatment of calves with bovine respiratory disease: Duration of therapy and posttreatment intervals. Vet. Clin. North Am. Food Anim. Pract. 31:441-453.

Arthington, J. D., C. A. Jaynes, H. D. Tyler, S. Kapil, and J. D Quigley 3rd.. 2002. The use of bovine serum protein as an oral support therapy following coronavirus challenge in calves. J. Dairy Sci. 85:1249-1254.

Bagwe, S., L. J. Tharappel, G. Kaur, and H. S. Buttar. 2015. Bovine colostrum: An emerging nutraceutical. J. Complement. Integr. Med. 12:175-185. https://doi.org/10.1515/jcim-2014-0039.

Berge, A. C., T. E. Besser, D. A. Moore, and W. M. Sischo. 2009. Evaluation of the effects of oral colostrum supplementation during the first fourteen days on the health and performance of preweaned calves. J. Dairy Sci. 92:286-295.

Berge, A. C., P. Lindeque, D. A. Moore, and W. M. Sischo. 2005. A clinical trial evaluating prophylactic and therapeutic antibiotic use on health and performance of preweaned calves. J. Dairy Sci. $88: 2166-2177$.

Blum, J. W., and H. Hammon. 2000. Colostrum effects on the gastrointestinal tract, and on nutritional, endocrine and metabolic parameters in neonatal calves. Livest. Prod. Sci. 66:151-159.

Chamorro, M. F., P. H. Walz, D. M. Haines, T. Passler, T. Earleywine, R. A. Palomares, K. P. Riddell, P. Galik, Y. Zhang, and M. D. Givens. 2014. Comparison of levels and duration of detection of antibodies to bovine viral diarrhea virus 1 , bovine viral diarrhea virus 2 , bovine respiratory syncytial virus, bovine herpesvirus 1 , 
and bovine parainfluenza virus 3 in calves fed maternal colostrum or a colostrum-replacement product. Can. J. Vet. Res. 78:81-88.

Chamorro, M. F., P. H. Walz, T. Passler, R. Palomares, B. W. Newcomer, K. P. Riddell, J. Gard, Y. Zhang, and P. Galik. 2016. Efficacy of four commercially available multivalent modified-live virus vaccines against clinical disease, viremia, and viral shedding in early-weaned beef calves exposed simultaneously to cattle persistently infected with bovine viral diarrhea virus and cattle acutely infected with bovine herpesvirus 1. Am. J. Vet. Res. 77:88-97.

Chantziaras, I., F. Boyen, B. Callens, and J. Dewulf. 2014. Correlation between veterinary antimicrobial use and antimicrobial resistance in food-producing animals: A report on seven countries. J. Antimicrob. Chemother. 69:827-834.

Chelack, B. J., P. S. Morley, and D. M. Haines. 1993. Evaluation of methods for dehydration of bovine colostrum for total replacement of normal colostrum in calves. Can. Vet. J. 34:407-412.

Cheng, G., H. Hao, S. Xie, X. Wang, M. Dai, L. Huang, and Z. Yuan. 2014. Antibiotic alternatives: The substitution of antibiotics in animal husbandry? Front. Microbiol. 5:217.

Dewell, R. D., L. L. Hungerford, J. E. Keen, W. W. Laegreid, D. D. Griffin, G. P. Rupp, and D. M. Grotelueschen. 2006. Association of neonatal serum immunoglobulin G1 concentration with health and performance in beef calves. J. Am. Vet. Med. Assoc. 228:914-921.

FASS. 2010. Guide for the Care and Use of Agricultural Animals in Research and Teaching. 3rd ed. Fed. Anim. Sci. Soc., Champaign, IL.

Fecteau, G., J. Paré, D. C. Van Metre, B. P. Smith, C. A. Holmberg, W. Guterbock, and S. Jang. 1997. Use of a clinical sepsis score for predicting bacteremia in neonatal dairy calves on a calf rearing farm. Can. Vet. J. 38:101-104

Furlund, C. B., A. B. Kristoffersen, T. G. Devold, G. E. Vegarud, and C. M. Jonassen. 2012. Bovine lactoferrin digested with human gastrointestinal enzymes inhibits replication of human echovirus 5 in cell culture. Nutr. Res. 32:503-513.

Hagiwara, K., S. Kataoka, H. Yamanaka, R. Kirisawa, and H. Iwai. 2000. Detection of cytokines in bovine colostrum. Vet. Immunol. Immunopathol. 76:183-190.

House, J. A. 1978. Economic impact of rotavirus and other neonatal disease agents of animals. J. Am. Vet. Med. Assoc. 173:573-576.

Klein-Jöbstl, D., M. Iwersen, and M. Drillich. 2014. Farm characteristics and calf management practices on dairy farms with and without diarrhea: A case-control study to investigate risk factors for calf diarrhea. J. Dairy Sci. 97:5110-5119.

Meganck, V., G. Hoflack, S. Piepers, and G. Opsomer. 2015. Evaluation of a protocol to reduce the incidence of neonatal calf diarrhoea on dairy herds. Prev. Vet. Med. 118:64-70.

Murray, C. F., L. J. Fick, E. A. Pajor, H. W. Barkema, M. D. Jelinski, and M. C. Windeyer. 2016. Calf management practices and associations with herd-level morbidity and mortality on beef cow-calf operations. Animal 10:468-477.

National Animal Health Monitoring System (NAHMS). 2007. Dairy 2007. Part 1: Reference of Dairy Health and Management in the United States USDA-APHIS Veterinary Services, Ft. Collins, CO.

Pardon, B., J. Alliët, R. Boone, S. Roelandt, B. Valgaeren, and P. Deprez. 2015. Prediction of respiratory disease and diarrhea in veal calves based on immunoglobulin levels and the serostatus for respiratory pathogens measured at arrival. Prev. Vet. Med. 120:169-176.

Parreño, V., G. Marcoppido, C. Vega, L. Garaicoechea, D. Rodriguez, L. Saif, and F. Fernández. 2010. Milk supplemented with immune colostrum: Protection against rotavirus diarrhea and modulatory effect on the systemic and mucosal antibody responses in calves experimentally challenged with bovine rotavirus. Vet. Immunol. Immunopathol. 136:12-27.

Pinchak, W. E., D. R. Tolleson, M. McCloy, L. J. Hunt, R. J. Gill, R. J. Ansley, and S. J. Bevers. 2004. Morbidity effects on productivity and profitability of stocker cattle grazing in the Southern Plains. J. Anim. Sci. 82:2773-2779.

Quigley, J. D., and T. M. Wolfe. 2003. Effects of spray-dried animal plasma in calf milk replacer on health and growth of dairy calves. J. Dairy Sci. 86:586-592.

Shearer, A. G. 1986. Internal navel abscesses in calves. Vet. Rec. 118:480-481.

Silbergeld, E. K., M. Davis, J. H. Leibler, and A. E. Peterson. 2008. One reservoir: Redefining the community origins of antimicrobialresistant infections. Med. Clin. North Am. 92:1391-1407.

Snodgrass, D. R., J. Stewart, J. Taylor, F. L. Krautil, and M. L. Smith. 1982. Diarrhoea in dairy calves reduced by feeding colostrum from cows vaccinated with rotavirus. Res. Vet. Sci. 32:70-73.

Sponseller, J. K., J. A. Steele, D. J. Schmidt, H. B. Kim, G. Beamer, X. Sun, and S. Tzipori. 2015. Hyperimmune bovine colostrum as a novel therapy to combat Clostridium difficile infection. J. Infect. Dis. 211:1334-1341.

Steele, J., J. Sponseller, D. Schmidt, O. Cohen, and S. Tzipori. 2013. Hyperimmune bovine colostrum for treatment of GI infections: A review and update on Clostridium difficile. Hum. Vaccin. Immunother. 9:1565-1568

Tyler, K. M., S. T. Normand, and N. J. Horton. 2011. The use and abuse of multiple outcomes in randomized controlled depression trials. Contemp. Clin. Trials 32:299-304.

US Food and Drug Administration. 2010. Congressional Testimony. Antibiotic Resistance and the Use of Antibiotics in Animal Agriculture. Accessed Aug. 23, 2016. http://www.fda.gov/ NewsEvents/Testimony/ucm219015.htm.

US Food and Drug Administration. 2015. FACT SHEET Veterinary Feed Directive Final Rule and Next Steps. Accessed Aug. 23, 2016. http://www.fda.gov/AnimalVeterinary/ DevelopmentApprovalProcess/ucm449019.htm.

Windeyer, M. C., K. E. Leslie, S. M. Godden, D. C. Hodgins, K. D. Lissemore, and S. J. LeBlanc. 2014. Factors associated with morbidity, mortality, and growth of dairy heifer calves up to 3 months of age. Prev. Vet. Med. 113:231-240.

Xie, G., G. C. Duff, L. W. Hall, J. D. Allen, C. D. Burrows, J. C. Bernal-Rigoli, S. E. Dowd, V. Guerriero, and C. J. Yeoman. 2013. Alteration of digestive tract microbiome in neonatal Holstein bull calves by bacitracin methylene disalicylate treatment and scours. J. Anim. Sci. 91:4984-4990. 